\title{
¿IMPERIALISMO DE LA ARGUMENTACIÓN? COMENTARIOS AL CURSO \\ DE ARGUMENTACIÓN JURÍDICA DE MANUEL ATIENZA
}

\author{
Mauro Barberis \\ Universidad de Trieste, Dep. IUSLIT
}

RESUMEN. El reciente Curso de argumentación jurídica, de Manuel ATIENZA, además de un manual de gran utilidad, es una poderosa reivindicación del espacio de la argumentación en la práctica y en la teoría del derecho. Esta reivindicación, no obstante, plantea el problema, no tematizado por el autor, de los límites conceptuales entre la argumentación y la interpretación: éste es el principal problema, si no el único, abordado en este trabajo. Comienzo examinando las relaciones entre los dos conceptos en el Curso; luego, redefino los límites entre éstos en términos más próximos al sentido ordinario de «interpretación» y «argumentación»; finalmente, critico lo que denomino el imperialismo de la argumentación de ATIENZA, reformulando la objeción de imperialismo de la moral ya planteada por otros y por mí.

Palabras clave: argumentación, interpretación, razonamiento jurídico, derecho, moral.

\section{Imperialism of Argumentation? Remarks on Manuel Atienza's Curso de argumentación jurídica}

ABSTRACT. The recent textbook of Manuel ATIENZA Curso de argumentación jurídica (2013) provides a powerful vindication of the space of argumentation both in practice and in theory of law. Such a vindication, however, raises the problem, not thematised by the Author, of the conceptual boundaries between interpretation and argumentation: in fact, the main, maybe the only question addressed to by this paper. I begin by examining the relationship between the concepts of interpretation and argumentation in the textbook; then, I define their boundaries in terms closer to ordinary language's senses; finally, I criticize what I call ATIENZA's imperialism of argumentation, an attitude analogous to imperialism of morals yet criticized by others and by me.

Keywords: argumentation, interpretation, legal reasoning, law, morals.

* Fecha de recepción: 14 de abril de 2014. Fecha de aceptación: 2 de julio de 2014. 
«Una metodología epistémica que

haga coincidir la búsqueda del conocimiento con la del poder [...] degrada innecesariamente el valor del conocimiento».

(A. SEN)

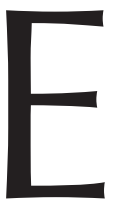

1 reciente Curso de argumentación jurídica, de Manuel ATIENZA, además de un manual de gran utilidad, es una poderosa reivindicación del espacio de la argumentación en la práctica y en la teoría del derecho ${ }^{1}$. Esta reivindicación, no obstante, plantea el problema, no tematizado por el autor, de los límites conceptuales entre la argumentación y la interpretación: éste es el principal problema, si no el único, abordado en este trabajo. Comienzo examinando las relaciones entre los dos conceptos en el Curso; luego, redefino los límites entre éstos en términos más próximos al sentido ordinario de «interpretación» y «argumentación»; finalmente, critico lo que denomino el imperialismo de la argumentación de ATIENZA, reformulando la vieja objeción de imperialismo de la moral.

\section{EL ESPACIO DE LA ARGUMENTACIÓN}

En toda teoría de la interpretación, de la argumentación y del razonamiento jurídico, los ingredientes principales son, en orden de importancia, definiciones, valoraciones y proposiciones. En este sentido, la teoría construida por ATIENZA en el Curso se podría representar así. El autor se propone, no simplemente analizar, sino redefinir los conceptos de interpretación, argumentación y razonamiento jurídico con el fin de integrarlos en una teoría del derecho predominantemente normativa, calificada ahora por él como pospositivista, pragmática o crítica ${ }^{2}$. El resultado es que el Curso absorbe en el concepto de argumentación no sólo a la interpretación, sino también al razonamiento jurídico.

Las preferencias teóricas de ATIENZA son obviamente legítimas, pero discutibles. En el derecho, por argumentación se entiende generalmente la actividad consistente en proporcionar argumentos a favor o en contra de una cierta atribución de significado —o sea, la argumentación de la interpretación — actividad que ciertamente no agota el razonamiento jurídico. ATIENZA, sin embargo, proporciona una redefinición latísima de argumentación o tal vez una definición estipulativa: la argumentación sería cualquier actividad discursiva relativa a un problema práctico, desde que se formula hasta que se resuelve mediante inferencias, que es además racional, en el doble sentido de instrumental a un fin pero también susceptible de valoración ética y no sólo técnica (C 109-110).

1 AtIENZA, 2013a, en adelante, se citará como C seguido del número de página.

2 ATIENZA, 2013a: 826: «Contribuir a la mejora del derecho y de las instituciones sociales [...] son, yo creo, las únicas finalidades que pueden dar sentido a la teoría del derecho; los propósitos descriptivos y explicativos tienen que estar al servicio de esos fines últimos». Estoy de acuerdo: los fines últimos de la teoría del derecho son prácticos. Pero es precisamente para conseguir esos fines prácticos que necesitamos de una teoría cognoscitiva y avalorativa, tal como en la física: si no pudiéramos confiar en el carácter cognoscitivo y avalorativo de las leyes de la mecánica, no podríamos usarlas para construir, por ejemplo. 
Una definición tan general de la argumentación, me parece, termina por coincidir con la de razonamiento jurídico e incluso con la de razonamiento ético o práctico, pero sobre todo restringe indebidamente el espacio de la interpretación. De hecho, un libro de casi novecientas páginas, riquísimo en ideas, sugerencias y materiales para profundizar, todos didáctica y científicamente valiosos, termina por dedicar expresamente a la interpretación sólo un apartado de doce páginas, ubicado en el sexto capítulo titulado «¿Cómo analizar las argumentaciones?» (C 513-525). Y digo «dedica expresamente» porque luego, en realidad, el libro no hace sino hablar de interpretación: pero, aclaro, lo hace casi siempre bajo el rótulo de argumentación.

Tampoco puede decirse que esta desproporción dependa sólo de la finalidad de promocionar la argumentación, objetivo al que la escuela de Alicante dedica un concurrido máster internacional ${ }^{3}$. Por el contrario, ATIENZA persigue el objetivo -anticipado en El derecho como argumentación (2006), donde ya distinguía las concepciones o dimensiones allí llamadas (C 104) formal, material y pragmática ${ }^{4}$ de la argumentación- de construir una teoría del derecho como argumentación en muchos sentidos análoga a la teoría interpretativa del derecho de Ronald DwORKIN. Se trata, en ambos casos, de teorías normativas, críticas y/o pragmáticas; sólo cambia, para mejor, la denominación, que en el caso de DwORKIN era particularmente equívoca.

ATIENZA redefine «interpretación» en el sentido que Jerzy WRÓBLEWSKY denomina estricto, por oposición al sentido lato. En sentido lato, «interpretación» designa la atribución de significado a cualquier texto, claro u oscuro, lo que vuelve «obvio que argumentar implica necesariamente interpretar». En cambio, la adopción del sentido estricto - atribución de significado a un texto oscuro- convierte «los problemas interpretativos» en «un subtipo de los problemas argumentativos» (C 514). La interpretación se convierte así en una de tantas argumentaciones o inferencias jurídicas, a la cual pueden dedicarse las pocas páginas ya mencionadas, destinadas originalmente a otro libro que ATIENZA iba a escribir con Isabel LifANTE (C 513, n. 25).

Tal vez convenga repetir que redefinir la interpretación como un subtipo de argumentación, modificando la relación tradicional entre los dos conceptos, es perfectamente lícito y ni siquiera revolucionario. Hace más de treinta años, por ejemplo, ya Aulis AARNIO, Robert AlEXY y Aleksander PECZENIK propusieron una importante redefinición del mismo tipo, formulándola no obstante en términos de razonamiento jurídico y no de argumentación ${ }^{5}$. Una tarea de esta magnitud, sin embargo, debería afrontar expresamente el concepto de interpretación y no deshacerse de él en pocas páginas, afirmando que «interpretación» es «una noción eminentemente polémica» (C 514), quizá en el sentido de essentially contested.

Llamo a este enfoque, que resume y enfatiza los extremos de la concepción de ATIENZA, imperialismo de la argumentación. Se trata de la tendencia a reducir a argumentación, no la teoría del derecho, sino el razonamiento jurídico, y sobre todo la interpretación, rebasando así los límites trazados tanto por los precursores de la teoría (Luis ReCASÉns Siches, Theodor WieHWEg, Chaïm PERELMAN), como por los partida-

\footnotetext{
3 Cfr. AtIEnZA, 2013b.

4 Cfr. ATIENZA, 2012.

5 Cfr. Aarnio, Alexy y Peczenik, 1987.
} 
rios de la teoría estándar de la argumentación jurídica (WRÓBLEWSKY, AARNIO, ALEXY, PECZENIK y Neil MACCORMick) ${ }^{6}$. Discutiré el imperialismo de la argumentación en la última sección, pero señalo inmediatamente que esta postura produce por lo menos cinco incongruencias.

Primera incongruencia. Ciertamente se puede elegir el sentido estricto de «interpretación», en homenaje a la vieja y equívoca máxima «in claris non fit interpretatio»; a su modo, también lo hace Paolo COMANDUCCI en un trabajo ya discutido en otra parte por ATIENZA, y parcialmente citado también en el Curso, donde el sentido de «interpretación» que WRÓBLEWSKY llama estricto fue rebautizado simplemente como jurídico ${ }^{7}$. COMANDUCCI, por otra parte, no excluía en modo alguno el sentido lato, entre otras razones porque, al hacerlo, todos los casos de interpretación serían oscuros por definición, y respecto de la interpretación así redefinida el escepticismo interpretativo se convertiría en analíticamente (y trivialmente) verdadero.

Segunda incongruencia. Sobre todo si se adopta el escepticismo interpretativo y se admite que todos los textos jurídicos pueden resultar oscuros, entonces la argumentación se convierte en un tema estratégico; es sólo con esta condición, de hecho, que se vuelve necesario justificar la atribución a un texto de uno entre sus diversos significados posibles. Históricamente, la interpretación se ha convertido en la actividad por antonomasia del jurista al menos desde que el derecho se basa en la escritura. La argumentación, sin embargo, ha sido tematizada más tarde, a partir de que el derecho fue codificado y existe la obligación de motivar las sentencias, una adquisición, esta última, aun más reciente en el common law que en el derecho continental ${ }^{8}$.

Tercera incongruencia. La obligación de motivar, hoy, se aplica a la interpretación de todos los textos, claros u oscuros, por lo que reducir la interpretación a una posibilidad que se actualiza sólo en el caso de textos oscuros ciertamente no favorece el desarrollo de una teoría de la argumentación. El adagio «in claris non fit interpretatio» ${ }^{9} \mathrm{im}$ plica también «in claris non fit argumentatio»; predicar claridad de un texto, de hecho, siempre ha servido para exentar de la obligación de justificar la interpretación elegida. Más en general, interpretación y argumentación son conceptos relacionados y correlativos, que conciernen a actividades estrechamente entrelazadas: ¿qué se gana, desde el punto de vista estrictamente cognoscitivo, al reducir cualquiera de ellos al otro?

Cuarta incongruencia. En el sentido latísimo que ATIENZA atribuye a «argumentación», toda interpretación es por definición argumentación: la solución de problemas prácticos extrayendo conclusiones a partir de las premisas. Si se adopta un sentido menos omnicomprensivo, en cambio, uno se da cuenta de que las relaciones entre los dos conceptos han sido siempre problemáticas. Por citar sólo un ejemplo: si no se considera argumentación la mera atribución a un texto de su significado supuestamente literal — como hace Giovanni TARELLO excluyendo la interpretación literal del propio elenco de los argumentos, como se menciona en el Curso- entonces bien puede darse

6 Cfr. ya en ATIENZA, 1991, y recientemente ATIENZA, 2013a: 31, donde objeta a la teoría estándar porque restringe «excesivamente su campo de estudio, en cuanto se ha centrado básicamente en las argumentaciones llevadas a cabo por los tribunales superiores y, por tanto, en los problemas de interpretación».

7 Cfr. C 130-135, y COMANDUCCI, 2011, comentado por ATIENZA en FERRER y RATTi, 2011: 71-79.

8 Cfr., al final, FrEDA, 2012.

9 Cfr. TARELlo, 1996: 67-69. 
interpretación sin argumentación: lo que resulta inconcebible, en cambio, adoptando el sentido de ATIENZA.

Quinta y última incongruencia. ¿Qué sentido tiene atribuirle a «argumentación» un significado tan amplio hasta hacerlo coextensivo de «razonamiento jurídico»? La pregunta no es retórica: en realidad, extender así el sentido de «argumentación» sirve realmente a un propósito: ciertamente no a reducir todo el derecho a argumentación - como oportunamente precisa ATIENZA varias veces- pero sí para hacer girar toda la práctica y la teoría del derecho en torno a la discusión sobre los valores. Ahora, yo también creo que la dimensión valorativa es esencial para una teoría del derecho en el Estado constitucional; ¿pero - y ésta en cambio sí es una pregunta retórica- es indispensable, para subrayarlo, extender indefinidamente el significado de «argumentación»?

\section{DELIMITACIÓN DEL CAMPO DE LA ARGUMENTACIÓN}

Para reconstruir de un modo más equilibrado las relaciones entre interpretación y argumentación, hago notar en primer lugar que, previamente a la distinción entre interpretación en sentido lato y en sentido estricto, à la WRÓBLEWSKY, hay al menos otra, tan común que es mencionada en un pasaje de Alf Ross citado en C 517, y que es usada por el mismo ATIENZA, aunque empleando la misma denominación usada para la anterior distinción ${ }^{10}$. En ausencia de una denominación consolidada, en mis trabajos distingo también entre interpretación en sentido genérico y en sentido específico. En sentido específico, «interpretación» sigue indicando cualquier atribución de significado: a textos oscuros (sentido estricto), a textos claros (sentido lato) o incluso a hechos (sentido latísimo).

En sentido genérico, en cambio, «interpretación» indica poco menos que la actividad entera de los juristas, o, más precisamente, su actividad intelectual o racional: esto es, el razonamiento jurídico, incluyendo la argumentación de la interpretación. En suma, la actividad discursiva y/o racional de los juristas - el razonamiento jurídico- se presta a ser representada, o quizá simplemente denominada, de dos modos opuestos. $\mathrm{O}$, como se usa en Génova — pero quizá no sólo allí- como interpretación en el sentido genérico, incluyendo la argumentación de la interpretación en sentido específico; o bien, como se usa en Alicante, como argumentación, en un sentido tan lato que abarque la interpretación en sentido específico.

En el análisis del lenguaje, como es sabido, los usos ordinarios son sólo la primera palabra, no la última; por otra parte, cualquier definición, si no quiere reducirse a una mera estipulación, deberá tener en cuenta los usos ordinarios de las palabras: y también, en este caso, el uso prevaleciente de «argumentación» como una parte de la interpretación en sentido genérico. Mi objetivo, por otro lado, no es por cierto defender los usos ordinarios ni, Dios nos libre, los usos genoveses contra los alicantinos, sino más bien el de trazar una distinción entre interpretación y argumentación que sea, empero,

${ }^{10}$ Cfr. AtIENZA, 2013a: 436: «El término “interpretación” ha de entenderse aquí en sentido estricto. En un sentido más amplio, cabría decir que todos los problemas normativos son problemas de interpretación». 
compatible con los usos ordinarios, pero también, y sobre todo, útil a finalidades ya sean cognoscitivas ya normativas.

Rechazando ambas tentaciones reduccionistas - la genovesa de reducir el razonamiento jurídico a interpretación, y la alicantina de reducirlo a argumentación- prefiero partir de dos significados más circunscritos de «interpretación» y de «argumentación». En cuanto a la interpretación, opto por el sentido específico de «interpretación»: la atribución de significado, en particular a textos ${ }^{11}$. En cuanto a la argumentación, sin embargo, prefiero partir del sentido ordinario de «argumentación», que por otra parte - y en esto tiene razón ATIENZA — hace tiempo que no está ya limitado a la argumentación de la interpretación, sino que se extiende a todas las inferencias que no son lógico-deductivas, y por tanto podría denominarse inferencial ${ }^{12}$.

En suma, entre el sentido de «argumentación» como argumentación de la interpretación, que puede llamarse mínimo, y aquel omnicomprensivo de ATIENZA, que puede llamarse máximo, hay uno intermedio, pero más próximo al máximo que al mínimo. En este tercer sentido, la argumentación comprende todos los razonamientos justificativos adicionales a la interpretación y a la deducción: los problemas de subsunción y calificación del hecho, incluyendo los temas de la aplicabilidad y de la derrotabilidad, la inferencia probatoria, inductiva o abductiva, por no hablar de toda la tópica, la retórica, la dialéctica... Un universo de temas, como se ve, apto para justificar suficientemente la ampliación del significado llevada a cabo por ATIENZA.

Siempre que «argumentación» no devenga sinónimo de «razonamiento jurídico» y absorba a la deducción y la interpretación, y mientras no permita olvidar distinciones importantes, extender generosamente el sentido del término es oportuno. El problema comienza cuando, como se hace en este trabajo, uno se pregunta dónde comienza la argumentación y dónde termina la interpretación. En el lenguaje ordinario, la delimitación entre los dos conceptos es elusiva; además, incluso después de las precisiones hechas aquí, es obvio que los sentidos (específico) de «interpretación»e (inferencial) de «argumentación» a menudo terminan por superponerse. El proble$\mathrm{ma}$, por otra parte, sigue siendo bastante importante, aunque siempre afrontado bajo otros rótulos.

Piénsese, en primer lugar, en distinciones como las existentes entre interpretación e integración (o construcción o creación), interpretación extensiva y analogía, norma explícita y norma implícita. Considérese la discusión sobre la interpretación constitucional, viciada precisamente por la confusión entre interpretación de la constitución, no demasiado disímil de la interpretación de la ley ordinaria, y argumentación constitucional, cuyos argumentos, como ponderación, proporcionalidad, razonabilidad y similares, sí son bastante diferentes de los argumentos empleados por los jueces ordinarios. Piénsese, por último, en el mismo tema de las relaciones entre el derecho y la moral: ¿en qué punto se deja de interpretar el derecho como es, y se comienza a cambiarlo por el derecho que se querría que fuese?

11 En rigor, puede hablarse de atribución de significado también a hechos (el sentido latísimo de WRÓBLEWSKI, la calificación del hecho) y a comportamientos (la interpretación de la costumbre).

12 Aunque en el sentido del inferencialismo semántico de Robert BRANDOM y, en Italia, de Damiano CANale y Giovanni Tuzet. 
Una discusión sobre muchos de estos problemas se ha celebrado hace poco en las páginas de la Rivista di filosofia del diritto, a propósito de un libro de Riccardo GuASTINI titulado precisamente Interpretare e argomentare (2011). Giorgio PINO y Enrico DiCIOTTI han puesto oportunamente en duda muchas de las (presuntas) dicotomías recién mencionadas, si no la misma oposición entre creación y aplicación del derecho ${ }^{13}$ que pretende abarcar y justificar todas las demás. Todo esto produce un bonito dilema, ya sea cognoscitivo ya normativo: ¿defender todas las pequeñas dicotomías, palmariamente vagas, con el único propósito de defender la gran oposición; o dejar que vayan colapsando las unas y la otra ${ }^{14}$ ?

Aquí me gustaría sostener que se puede trazar una distinción conceptual entre interpretación y argumentación que pasa entre aquellos que TARELLO llamaba usos interpretativos y usos creativos de la argumentación, distinción que emerge también de la discusión recién señalada. En su réplica, de hecho, GuASTINI admite que entre normas expresas, fruto de la interpretación, y normas no expresas, fruto de la argumentación, los confines son lábiles: «no existe una clara distinción entre eso que un texto significa y eso que su significado implica» ${ }^{15} \mathrm{o}$ — diría más bien- eso que de su significado se puede inferir. Sin embargo, la distinción está precisamente aquí: radica entre la atribución de significado a una disposición, y las inferencias que de tal significado se pueden extraer.

La distinción entre lo que un texto significa (interpretación) y lo que se puede inferir de él (argumentación) es fijada — en cada instante, aunque en modo cambiante entre momento y momento- a partir del uso consolidado por los juristas y, en última instancia, por los órganos de aplicación. Pongamos que el art. 48 de la Constitución italiana, «Son electores todos los ciudadanos» sea hoy comúnmente interpretado a contrario, en el sentido de que los no ciudadanos no son electores: bien, ésta es, hoy, la norma explícita, la interpretación de la disposición. Las otras interpretaciones, como la literal y la analógica (el art. 48 nada diría sobre los no ciudadanos, habría aquí una laguna colmable por analogía) producen hoy, en cambio normas no expresas y fruto de la argumentación: aunque mañana podrían convertirse en normas explícitas, producto de la interpretación.

\section{3. ¿IMPERIALISMO DE LA ARGUMENTACIÓN?}

Hay fuertes razones cognoscitivas, como se ha visto, para extender el significado de «argumentación». La ampliación del significado llevada a cabo en el Curso, por otra parte, está justificada sobre todo en razones normativas, lo que resumo bajo la rúbrica de imperialismo de la argumentación: la posición actual de ATIENZA, en otros términos, parece una sofisticada reformulación de una posición criticada por otros y por mí como imperialismo de la moral. Carlos NiNO, actualmente el mayor punto

13 Cfr. Guastini, 2011 y 2013; DiciotTi, 2013; PinO, 2013.

14 Defender las pequeñas dicotomías para que no vaya a colapsar la grande es la estrategia (política) declarada en VILLA, 2011: otro autor pragmatista que ha derivado explícitamente las elecciones definitorias de sus preferencias normativas, exactamente como ATIENZA.

15 Así en Guastini, 2013: 135. 
de referencia de ATIENZA, escribía en su póstumo Derecho, moral y política (1994) que «el discurso moral de la modernidad tiene un carácter imperialista que impide la subsistencia de discursos justificativos insulares». Entonces, el derecho no justificaría acciones o decisiones a menos que sea conforme con la moral ${ }^{16}$.

A esta posición, y más en general, a la Tesis de la unidad del razonamiento prácti$\mathrm{Co}^{17}$, Cristina REDONDO y el suscrito reprochábamos, por razones diversas, subordinar el derecho a la moral instituyendo una triple conexión necesaria —-definitoria, justificativa e interpretativa- unidireccionalmente de la moral al derecho ${ }^{18}$. La necesidad de tal conexión, especialmente en el razonamiento judicial, es dudosa: el juez está obligado a motivar las propias decisiones sobre la base del derecho, y no de la moral ${ }^{19}$. COMANDUCCI, en el trabajo ya citado y parcialmente referido en el Curso, sostenía que tal necesidad es meramente definitoria: depende de la definición de «razón última» como razón moral implícitamente adoptada por NiNO (C 132).

Todos los participantes en la discusión desde hace veinte años hasta hoy sostienen posiciones más articuladas: y también lo hace ATIENZA. Rechazando una vez más la acusación de imperialismo de la moral, de hecho, él admite adoptar, hoy, una posición que deja mayor espacio a las razones jurídicas institucionales - las razones del derecho como institución - respecto de las razones morales. El razonamiento jurídico, para él, adopta una racionalidad del second best, que no persigue más la mejor solución moral, sino la mejor solución jurídica compatible con la moral ${ }^{20}$. ATIENZA, en otros términos, adopta el modelo de justificación en dos niveles propuesto por NiNO en Fundamentos de derecho constitucional (1992), cuya parte crucial, referida en el Curso, puede sintetizarse así:

[Frente al riesgo de una concepción del razonamiento jurídico justificativo que, al basarse en la moral, convierta en superflua la Constitución y el resto del ordenamiento jurídico] [...] creo que la solución está dada por un tipo de razonamiento escalonado en dos niveles: a) En el nivel primero y más básico se deben articular las razones que legitiman o no la práctica social constituida por la presente Constitución [...]. Pero en este primer nivel del razonamiento práctico se deben tener en cuenta cuáles son las alternativas realistas a la preservación de la presente Constitución. Es posible que, si bien ella esté bastante distante del punto óptimo de legitimidad, [...] cualquier alternativa realista esté todavía más alejada [...]. b) Si el resultado del razonamiento del primer nivel es favorable a la legitimidad, aún imperfecta, de la presente Constitución, es posible pasar al segundo nivel, en que se trata de aplicar esa Constitución para justificar acciones o decisiones (C 359).

Ante la decisión de un caso, entonces, el juez debe preguntarse, primero, si la constitución vigente es moralmente legítima aunque existan alternativas realistas a ella; y dado que casi siempre tales alternativas son aleatorias, habitualmente el juez deci-

16 NiNO, 1999: 67.

17 Cfr. al final Dworkin, 2013. En el debate metaético actual, esta posición se denomina monismo, por oposición al pluralismo de los valores, pero creo que puede reinterpretarse caritativamente como una suerte de principio de indivisibilidad de todos los valores, un poco como en el Preámbulo de la Carta de Niza (2000): los valores se tienen en cuenta en su totalidad, no separadamente.

18 Cfr. RedONDO, 1998: esp. 365, y desde el título BARBERIS, 2003.

19 Cfr. Navarro y Redondo, 1994: 77.

20 Cfr. AtienZa, 2013a: 357, donde, refiriéndose a NiNO, observa que «la moral no juega, por ello, un papel "imperialista”, puesto que no puede imponer siempre lo que sería, en abstracto, la mejor solución (NINO) habla, por tanto, de la racionalidad jurídica como "de una racionalidad de lo segundo mejor"». 
dirá el caso sobre la base del derecho fundado en la misma constitución: las razones institucionales, así, normalmente terminan por prevalecer sobre las razones morales. La moral sigue siendo interpelada primero, pero el juez, en ausencia de alternativas, decide casi siempre sobre la base del derecho. Surge en efecto la pregunta de si alguna vez podría negarse a hacerlo así, porque está atenazado entre la acusación de denegación de justicia y la dimisión a la magistratura ${ }^{21}$; no obstante, como veíamos hace un momento, hay una alternativa: se llama interpretación.

El modelo de dos niveles que ATIENZA retoma de NINO es menos vulnerable a la acusación de imperialismo de la moral que las posiciones precedentes: el papel que allí juega la moral, en efecto, es máximo en teoría, pero mínimo en la práctica. De hecho, la moral juega quizá un papel mayor en la teoría de la argumentación de Jürgen HABERMAS, apoyada en la Tesis de la complementariedad entre el derecho y la moral: Tesis que se sustrae a la acusación de imperialismo de la moral, no obstante, precisamente porque no subordina en modo alguno el derecho a la moral, sino que les asigna funciones diferentes, autónomas y complementarias ${ }^{22}$. La teoría de NiNO-ATIENZA es sin embargo todavía pasible de otra crítica, no más en términos de imperialismo de la moral sino de imperialismo de la argumentación.

El modelo de NiNO-ATIENZA sigue sujeto a la objeción de imperialismo de la moral porque continúa subordinando el derecho a la moral: como si una solución moral, dictada por una moral positiva o una crítica, fuese siempre, por definición, mejor que una solución jurídica, lo que podría ser cuestionado al menos por los positivistas éticos o normativos. El modelo de NiNO-ATIENZA, además, se expone a la objeción de imperialismo de la argumentación: ciertamente, sea de hecho o de derecho, privilegia la argumentación (moral) respecto de la interpretación (jurídica). ¿Pero, de hecho, el juez se preguntará realmente si hay alternativas realistas a la constitución vigente, como querrían NiNO y ATIENZA, o más bien tratará de reinterpretar la constitución o disposición injustas? ¿Y, jurídicamente, qué otra cosa podría hacer?

Quizá, el modelo de justificación en dos niveles, pionero en la teoría del razonamiento jurídico ${ }^{23}$, podría reformularse de un modo más respetuoso de la distinción entre reglas y principios, aceptada también por ATIENZA, y de la distinción entre interpretación y argumentación esbozada aquí. El primer nivel se ocuparía de los principios constitucionales, como formulaciones jurídicas de valores morales o más en general éticos; en el Estado constitucional, en efecto, cualesquiera que sean el tipo de rigidez de la constitución y los sistemas de control de legitimidad constitucional adoptados, la primera pregunta que el aplicador se plantea o debería plantearse es precisamente

21 Es probable que aquí NINO pensase en casos del tipo del derecho nazi, y no en el caso estándar de la aplicación del derecho en el Estado constitucional. En cualquier caso, si se acepta la crítica del fundacionalismo conocida como el argumento del Trilema de Münchhausen, propuesta por Hans ALBERT, parece problemático hablar de una legitimidad política o moral del sistema jurídico como un todo, distinta de la legitimidad constitucional: $c f r$. BARBERIS, 2014: 165-171.

22 Cfr. HABERMAS, 1996: 130: «Il diritto positivo porta sempre dentro di sè un incancellabile riferimento alla morale. Ma questo riferimento alla morale non deve indurci a subordinare il diritto alla morale nel senso di una gerarchia tra norme [...] Piuttosto, la morale autonoma da un lato e il diritto positivo (sempre bisognoso di fondazione) dall'altro si collocano in un rapporto di complementarità».

23 El modelo de justificación en dos niveles se remonta al menos al rule o restricted utilitarianism, y por tanto al razonamiento moral, pero ha sido aplicado al razonamiento judicial al menos desde WASSERSTROM, 1961. 
ésta: ¿la disposición a aplicar es conforme con la constitución, en una u otra de sus interpretaciones posibles?

El segundo nivel justificativo, por otra parte, se ocuparía de las reglas, aplicables sobre la base de las decisiones hechas en el primer nivel, interpretando, aplicando o integrando las disposiciones relevantes. Esquemáticamente, en el primer nivel, se argumenta más que interpreta, y en el segundo, se interpreta más que argumenta; entre los dos niveles, un papel decisivo es jugado por la así llamada interpretación conforme: argumento de la interpretación constitucional que puede asumir funciones tanto interpretativas como creativas. Pero la crítica moral del derecho sigue cumpliendo una función, con base en la Tesis de la complementariedad: aunque en el Estado constitucional, ciertamente, no todos los valores morales devengan internos al ordenamiento.

En el Dialogo que concluye el Curso el mismo ATIENZA califica la relación derechomoral en términos no más de medio a fin, sino de parte respecto del todo ${ }^{24}$; lejos de ser sólo una técnica al servicio de la moral, el derecho podría considerarse en sí mismo un valor: interpretación y argumentación, reglas jurídicas y principios constitucionales tendrían entonces funciones complementarias e indivisibles. En la página final del Curso, en efecto, ATIENZA escribe que: «El Derecho no son sólo actos intencionales y los resultados buscados con esos actos, sino una institución de la cual forman parte elementos que nunca han sido queridos por nadie, que están por encima de las conciencias individuales» (C 831). Concuerdo plenamente ${ }^{25}$.

(Traducción de Alejandro González Piña)

\section{REFERENCIAS BIBLIOGRÁFICAS}

Aarnio, A.; AleXy, R., y PeCZENIK, A., 1987: «I fondamenti del ragionamento giuridico», en P. COMANDUCCI y R. GUASTINI (a cura di), L'analisi del ragionamento giuridico, Torino: Giappichelli, 1987, 121-187, trad. it. parcial de R. GUASTINI $i d$., «The Foundation of Legal Reasoning», en Rechtstheorie, 12, 1981, 133-158, 257-279, 423-448.

ATIENZA, M., 1991: Las razones del derecho. Teorías de la argumentación jurídica, Madrid: Centro de Estudios Constitucionales.

- 2012: Diritto come argomentazione. Concezioni dell'argomentazione, trad. it. de V. NITRATO Izzo, Napoli: Editoriale scientifica, El derecho como argumentación. Concepciones de la argumentación, Barcelona: Ariel, 2006.

- 2013a: Curso de argumentación jurídica, Madrid: Trotta.

- 2013b: «Constitución y enseñanza de la argumentación jurídica», prólogo de P. GRÁNDEZ y F. Morales (eds.), La argumentación jurídica en el Estado constitucional, Lima: Palestra, 15-18.

BARBERIS, M., 2003: «Neoconstitucionalismo, democracia y imperialismo de la moral», en M. CARBOnell (ed.), Neoconstitucionalismo(s), Madrid: Trotta, 259-281, trad. cast. de S. SASTRE, «Neo-costituzionalismo, democrazia e imperialismo della morale», en Ragion pratica, 14, 2000, 147-162.

${ }^{24}$ Literalmente: «En fin, además de la relación medio-fin, creo que habría que considerar la relación parte-todo para dar adecuadamente cuenta del valor del derecho» (ATIENZA, 2013a: 831).

25 Cfr. BARBERIS, 2013. 
- 2013: «Los derechos humanos como adquisición evolutiva», en Doxa, 36, 25-40.

- 2014: Introduzione allo studio del diritto, Torino: Giappichelli.

COMAnducci, P., 2011: «Sobre la interpretación jurídica», en J. FerRer Beltrán y G. B. RATTI (eds.), El realismo jurídico genovés, Madrid: Marcial Pons, 51-70.

Dworkin, R., 2013: Giustizia per i ricci, Milano: Feltrinelli, trad. it. de V. OTTONELLI, Justice for Hedgehoods, Cambridge (Mass.): Harvard U. P., 2011.

DiciotTi, E., 2013: «Norme espresse e norme inespresse. Sulla teoria dell'interpretazione di Riccardo Guastini», en Rivista di filosofia del diritto, 103-123.

FREDA, D., 2012: «Una dispotica creazione». Il precedente vincolante nella cultura giuridica inglese dell'Ottocento, Torino: Giappichelli.

GuASTINI, R., 2011: Interpretare e argomentare, Milano: Giuffrè.

- 2013: «Replica», en Rivista di filosofia del diritto, 1, 125-136.

Habermas, J., 1996: Fatti e norme, Milano: Guerini, trad. it. de L. CePPA, Faktizität und Geltung, Frankfurt: Suhrkamp, 1992.

NAVARRO, P., y REDONDO, C., 1994: Normas y actitudes normativas, México: Fontamara.

NinO, C. S., 1999: Diritto come morale applicata, Milano: Giuffrè, trad. it. di M. LA TORRE, Derecho, moral y política, Barcelona: Ariel, 1994.

PINO, G., 2013: «Interpretazione cognitiva, interpretazione decisoria, interpretazione creativa», en Rivista di filosofia del diritto, 1, 77-101.

REDONDO, C., 1988: «El carácter práctico de los deberes jurídicos», en Doxa, 21, 1998, vol. 2, 355-370.

TARELLO, G., 1976: Storia della cultura giuridica moderna, vol. 1: Assolutismo e codificazione del diritto, Bologna: Il Mulino.

VILlA, V., 2011: Una teoria pragmaticamente orientata dell'interpretazione giuridica, Torino: Giappichelli.

WASSERSTROM, R. A., 1961: The Judicial Decision: toward a Theory of Legal Justification, Stanford (Cal.): Stanford U. P. 